\title{
Incidence and linguistic quality of speech errors: a comparison of preoperative transcranial magnetic stimulation and intraoperative direct cortex stimulation
}

\author{
Ina Bährend, MD, ${ }^{1}$ Max R. Muench, ${ }^{1}$ Heike Schneider, ${ }^{1}$ Rabih Moshourab, MD, ${ }^{2}$ \\ Felix R. Dreyer, MD, ${ }^{3}$ Peter Vajkoczy, MD, ${ }^{1}$ Thomas Picht, MD, ${ }^{1}$ and Katharina Faust, MD' \\ Departments of ${ }^{1}$ Neurosurgery and ${ }^{2}$ Anesthesiology, Charité, Universitätsmedizin Berlin; and ${ }^{3}$ Brain Language Laboratory, \\ Freie Universität Berlin, Germany
}

\begin{abstract}
OBJECTIVE Given the interindividual variance of functional language anatomy, risk prediction based merely on anatomical data is insufficient in language area-related brain tumor surgery, suggesting the need for direct cortical and subcortical mapping during awake surgery. Reliable, noninvasive preoperative methods of language localization hold the potential for reducing the necessity for awake procedures and may improve patient counseling and surgical planning. Repetitive navigated transcranial magnetic stimulation (rnTMS) is an evolving tool for localizing language-eloquent areas. The aim of this study was to investigate the reliability of rnTMS in locating cortical language sites.
\end{abstract}

METHODS Twenty-five patients with brain tumors in speech-related areas were prospectively evaluated with preoperative rnTMS $(5 \mathrm{~Hz}$, train of five, average $105 \%$ resting motor threshold) and navigated direct cortical stimulation (DCS; bipolar, $50 \mathrm{~Hz}, 6-8 \mathrm{~mA}, 200$ - $\mu$ sec pulse width) during awake surgeries employing a picture-naming task. Positive and negative stimulation spots within the craniotomy were documented in the same MRI data set. TMS and DCS languagepositive areas were compared with regard to their spatial overlap, their allocation in a cortical parcellation system, and their linguistic qualities.

RESULTS There were over twofold more positive language spots within the exposed area on rnTMS than on DCS. The comparison of positive rnTMS and DCS (ground truth) overlaps revealed low sensitivity $(35 \%)$ and low positive predictive value $(16 \%)$ but high specificity $(90 \%)$ and high negative predictive value $(96 \%)$. Within the overlaps, there was no correlation in error quality. On DCS, $73 \%$ of language-positive spots were located in the pars opercularis and pars triangularis of the frontal operculum and $24 \%$ within the supramarginal gyrus and dorsal portion of the superior temporal gyrus, while on rnTMS language positivity was distributed more evenly over a large number of gyri.

CONCLUSIONS The current protocol for rnTMS for language mapping identified language-negative sites with good dependability but was unable to reliably detect language-positive spots. Further refinements of the technique will be needed to establish rnTMS language mapping as a useful clinical tool.

https://thejns.org/doi/abs/10.3171/2020.3.JNS193085

KEYWORDS functional mapping; language; navigated transcranial magnetic stimulation; direct cortical stimulation; glioma surgery; oncology; surgical technique

$\mathrm{T}$ RANSCRANIAL magnetic stimulation (TMS) has evolved as a powerful tool for preoperative functional mapping. The application of TMS in the motor system has led to refined preoperative risk assessments and improved postoperative results. ${ }^{1-5}$ Repetitive navigated TMS (rnTMS) combines conventional TMS technology with a navigation system that allows precise anatomical representation of functional areas within 3D brain reconstructions. ${ }^{6,7}$
The use of rnTMS has recently been extended to other neurological functions, ${ }^{8-10}$ notably in preoperative speech mapping. ${ }^{11,12}$ Some have even attempted to use rnTMS as the sole method of localizing language-eloquent areas prior to tumor resection, refraining from direct cortical stimulation (DCS) completely..$^{13}$ To evaluate and possibly improve current TMS protocols for speech mapping, the results of rnTMS use must be compared to those obtained with the current gold standard, which is DCS during awake sur-

ABBREVIATIONS aSMG = anterior supramarginal gyrus; DCS = direct cortical stimulation; IPI = interpicture interval; LR = likelihood ratio; MPRAGE = magnetization-prepared rapid gradient echo; NPV = negative predictive value; opIFG = opercular inferior frontal gyrus; PPV = positive predictive value; RMT = resting motor threshold; rnTMS = repetitive navigated TMS; TMS = transcranial magnetic stimulation; trlFG = triangular inferior frontal gyrus; vPrG = ventral precentral gyrus.

SUBMITTED December 27, 2019. ACCEPTED March 30, 2020.

INCLUDE WHEN CITING Published online May 29, 2020; DOI: 10.3171/2020.3.JNS193085. 
gery. ${ }^{14,15}$ Because awake brain surgery can be stressful for any patient and can be associated with certain procedurerelated risks, ${ }^{16,17}$ the reliability of methods of noninvasive preoperative speech allocation such as rnTMS should be improved to possibly reduce the number of necessary awake procedures, to lower the risk profile in patients who are ineligible for awake procedures, and to refine risk assessments to enable better patient counseling. Reliable noninvasive mapping protocols for language would also be useful for basic and translational neuroscience.

TMS and DCS share a common electrophysiological basis ${ }^{18,19}$ in which language inhibition is mediated by the induction of an electrical field that is assumed to be confined primarily to the cortical level. Both techniques are lesion based, with TMS offering the advantage of noninvasiveness. To date, the question of how far current density and electrical-field distribution can be compared between TMS and DCS remains unclear. ${ }^{20-22}$

Earlier feasibility reports by our group and by others $^{11,12,23}$ have produced varying results on rnTMS's degree of reliability in speech mapping. Tarapore et al. have reported promising results for rnTMS compared to the gold standard of DCS, specifying a sensitivity of $90 \%$ and a specificity of $98 \% .{ }^{11}$ An earlier two-center study, to which our group contributed, ${ }^{12}$ found comparably high sensitivity $(90 \%)$ but low specificity $(24 \%)$. Ille et al. ${ }^{23}$ have also reported high sensitivity $(100 \%)$ and low specificity $(5 \%)$. Overall, the rnTMS-based allocation of speech-eloquent areas has not comprehensively entered everyday clinical practice. In our own clinical experience, we have continuously experienced difficulties in reliably localizing language-positive spots with rnTMS when employing the protocols referenced above; indeed, we have failed to localize the language-dominant hemisphere with certainty. After gaining 8 years of experience in language rnTMS mapping in order to standardize protocols, we reassessed the validity of the method, this time with the supervision of a designated linguist. Our aim in the present study was to compare the current rnTMS protocol for language with a well-established DCS protocol during awake surgery. Preoperative TMS-positive spots were compared to intraoperative DCS-positive spots in terms of spatial overlaps and their affiliations within a cortical parcellation system, while also taking into account different qualities of speech errors.

\section{Methods \\ Ethics}

The study protocol was approved by the local ethics committee (Landesamt fuer Gesundheit und Soziales [LAGeSo] Berlin), in accordance with the Declaration of Helsinki. Informed consent was obtained from all study participants.

\section{Patients}

\section{Patient Selection}

Twenty-five right-handed patients with infiltratively growing tumors near presumed cortical language areas were prospectively enrolled in this study. Inclusion criteria were a scheduled awake surgery and a tumor in the domi- nant left perisylvian region. The exclusion criteria were 1) an age younger than 18 years, 2) an inability to provide informed consent, 3) a seizure frequency of $>1$ per week, 4) a pacemaker or metal device near the cranial area, 5) preexisting moderate or severe aphasia, and 6) compromised cognitive function.

\section{Language Assessment}

All patients were assessed with the DemTect (DS) test ${ }^{24}$ and subtests for verbal repetition, object naming, language comprehension, and the Token test from the Germanlanguage Aachen Aphasia Test (AAT). ${ }^{25}$ Patients who expressed moderate or severe aphasia according to their AAT results, as well as patients with a DS score $<9$, were excluded from the study.

For TMS and DCS mapping, we used a picture-naming task that consisted of 80 black-and-white drawings of everyday objects. The picture data set was previously controlled for consistent naming of $>90 \%$ in an in-house study with 28 mixed-age healthy volunteers. Before mapping, all patients performed three baseline runs with 80 images from the picture-naming tasks, in which pictures that were inconsistently named between runs by a patient were excluded to generate individual patient baseline sets. The same remaining objects of the baseline set were also later used for intraoperative baseline and mapping during DCS. Patients who could not name more than 58 of the presented pictures were also excluded. ${ }^{26}$

\section{Magnetic Resonance Imaging}

All patients underwent MRI in a 3-T (Siemens Vida) or 1.5-T (Siemens Magnetom Avanto) scanner. Contrastenhanced, 1-mm slice thickness magnetization-prepared rapid gradient echo (MPRAGE) images served as the basis for both cranial navigation and rnTMS.

\section{rnTMS Protocol}

The eXimia NBS version 3.2.2 and Nexstim NBS 4.3 with a NexSpeech module (Nexstim Oy) were used for the rnTMS mapping. The acquired MPRAGE sequence was imported into the TMS software to generate a 3D model of the brain and was then coregistered with the patient's skull anatomy.

A tracking camera was used to locate the coil position in relation to the patient's head; the induced electric field was then visualized within the local anatomy. ${ }^{6,12}$ To estimate the stimulation intensity needed to inhibit speech function, the resting motor threshold (RMT) was determined, as described elsewhere. ${ }^{27}$ Mapping commenced at $100 \%$ of each patient's individual RMT given that the induced electrical field was $>50 \mathrm{~V} / \mathrm{m}$. In field strengths $<50$ $\mathrm{V} / \mathrm{m}$, the RMT was increased to $110 \%$ or $120 \%$. If the patient felt discomfort, the intensity was reduced to $90 \%$ or $80 \%$ RMT. ${ }^{12}$ The average intensity was $105 \%$ RMT. The coil was aligned perpendicular to the most adjacent sulcus to provide optimum field induction. ${ }^{28}$ Impulses were applied as single bursts of five pulses at a frequency of $5 \mathrm{~Hz}$. The rnTMS burst initiation was time locked to the onset of object presentation, with a 0 -msec delay. The default interpicture interval (IPI) was 2.5 seconds. If indicated, the IPI was adjusted in 200-msec steps to accommodate 
individual performance. During the IPI, the stimulation coil was moved randomly to the next stimulation spot within a grid of a 1-cm interstimulus distance to cover the entire perisylvian area. Every spot was addressed at least three times. All rnTMS investigations were performed by a TMS technician with over 8 years of experience in language TMS mapping and were video recorded for repeat offline language analysis. The first analysis was conducted by the TMS technician and the second by a board-certified linguist. In cases of disagreement, the linguist's judgment was decisive.

Each positive rnTMS point was exported as a DICOM file with a depth of $25 \mathrm{~mm}$ into the navigation software (Brainlab Elements) and then color-coded according to the respective quality of error, for postoperative comparison with DCS spots.

\section{Surgical Workflow}

All surgeries were performed with the patient under local anesthesia and with the aid of low-dose systemic analgesia and sedation (propofol and remifentanil $2.5 \mathrm{mg} /$ $\mathrm{kg} / \mathrm{hr}$ ) during skull opening and wound closure. A scalp block was applied using bupivacaine mixed with epinephrine (ratio 250,000:1). The awake patients situated themselves into a comfortable position on the operating room table in a manner that allowed them a sufficient view of the display screen for object naming while simultaneously providing optimum tumor access to the operating surgeon. This generally involved a $60^{\circ}$ head tilt. Patients were fixed into a Mayfield head clamp. The skull surface was registered into navigation software (Brainlab Elements). Navigated craniotomies were performed in a manner corresponding to the size and location of each respective tumor, yet always large enough to provide an additional ample tumor-free cortical seam around the tumor bed for mapping. The size of each craniotomy was measured (area in $\mathrm{cm}^{2}$ ). Before awake testing, any intravenous analgesia and sedation had been stopped for a minimum of 15 minutes. Cortical mapping was completed before the corticotomy commenced.

\section{DCS Protocol}

Language mapping was initiated at least 15 minutes after propofol and remifentanil use had been completely suspended. At this time, patients had a Ramsay sedation score of 2 (patient cooperative, oriented, and calm).$^{29}$ Only after three consecutive baseline runs of the patient's individual picture-naming task was DCS initiated.

A bipolar single-use stimulation probe connected to the navigation pointer and operated through the ISIS intraoperative neuromonitoring system (Inomed Medizintechnik $\mathrm{GmbH}$ ) was used. The stimulation parameters were constant current, $0.2-\mathrm{msec}$ pulse width, $50-\mathrm{Hz}$ frequency, and 6- to $8-\mathrm{mA}$ stimulation intensity, with a pole distance of $10 \mathrm{~mm}$.

Effective functioning of the stimulation probe and adequate stimulation intensity were confirmed by evoking dysarthria in the central operculum, that is, the lowest suprasylvian part of M1 that encodes for the pharynx and tongue. The central operculum was exposed in all cases and easily identified based on anatomical landmarks. Dysarthria could be evoked in all cases with a stimulation intensity maximum of $8 \mathrm{~mA}$. The same stimulation intensity required to induce dysarthria was subsequently used for speech mapping in each respective patient (6-8 mA).

The entire area of the dural opening was mapped without gaps, corresponding to a map grid of a $1-\mathrm{cm}$ interstimulation distance. The "two out of three" rule was used, as described in earlier research..$^{30}$ Each stimulation point was stimulated three times and was scored positively if at least two of the three stimulations resulted in the same error quality. As was the case with our DCS protocol, however, all positive DCS spots resulted in the same error quality during all three runs, suggesting a 100\% reproducibility. Each stimulation-positive point, as localized by the navigation pointer, was immediately marked in the patient's MPRAGE sequence using the iPlan "acquire point" function.

During DCS, electroencephalography was recorded via a six-contact strip electrode to confirm that DCS was functional and to anticipate possible epileptic seizures.

TMS mapping data were not displayed in the navigation used during surgery. Thus, the surgeon who performed DCS stimulation, as well as all operating room personnel who participated in the generation and documentation of the DCS maps, was blinded to the TMS data. The quality of error for the acquired points was noted both by the electrophysiology technician and by a second auxiliary person. The DCS mappings were video recorded to enable retrospective analyses by the linguist to confirm the correct quality of error and to avoid confusion with possible speech disturbances attributable to other factors (e.g., temporary patient discomfort). In cases of inconsistencies, the linguist's post hoc analysis was decisive.

\section{Linguistic Quality of Error}

Occurring speech deficits were assigned to six error categories: 1) anomia/no response, complete absence of a verbal response; 2) semantic, correctly uttered misnomers, often semantically related to the actual target word (e.g., an egg was called a chicken); 3) phonological, incorrect use or exchange of phonemes within the target word (e.g., "tible" rather than "table"); 4) performance, errors in articulation of the response (i.e., the patient showed a dysarthria, e.g., "a-a-a-apple") during stimulation; 5) hesitation, a notable delay in giving a response, which could also have been introduced by an expletive word (e.g., "hmmmm..., ah..., wheelchair"); and 6) nonclassified, for example, in 2 patients, the error qualities during DCS could not be tracked reliably because of insufficient error documentation during DCS and deficient video sound recording.

\section{Comparison of rnTMS- and DCS-Positive Sites}

Analyses of TMS and DCS copositive points were done in each subject separately by two independent investigators. Two different algorithms were used to assess copositivity. With the first method, sites were defined as copositive if their distance was $<10 \mathrm{~mm}$. For counting, rnTMS- and DCS-positive spots were increased to spheres of $10 \mathrm{~mm}$ in a strictly sagittal 3D projection of 
the MPRAGE sequence to correct for deviations in depth. Those TMS spheres showing an overlap with DCS spheres were counted as true positives (Fig. 1). The rationale behind the $10-\mathrm{mm}$ sphere size was based on electrophysiology: the effective electrical field in DCS was assumed to lie between the two poles of the bipolar probe, ${ }^{31}$ the distance of which was $10 \mathrm{~mm}$ in our study. The cortical area activated by TMS was assumed to be of a similar size. ${ }^{11}$

In the second method, a gyral anatomy-based parcellation system was used, as described by Corina et al. ${ }^{32}$ The system has been favored in many TMS studies..$^{12,23}$ The cortex was parcellated into 37 distinct regions believed to constitute functional and connectivity-based entities (Fig. 2). In practice, this universal parcellation matrix was aligned to each patient's individual cortical contours based on their 3D MRI scans. Any DCS- and rnTMS-positive spots that fell into the same anatomical segment were registered as copositive. Analyses were again conducted on all spots, first regardless of the error type and then with respect to their specific speech quality.

Only TMS spots located within the area of dural opening were considered in the analysis. The area of dural opening was calculated as $\pi \mathrm{ab}$, with the two semi-axes of durotomy corresponding to the semi-axes of craniotomy minus $1 \mathrm{~cm}$. In cases of disparity between the investigators, the respective spot was jointly reevaluated a third time.

\section{Results}

\section{General Patient Characteristics and Tumor Distribution}

Twenty-five patients (11 female, 14 male) with glioma in speech-eloquent areas on the left hemisphere were operated on under awake craniotomy with DCS after preoperative rnTMS speech mapping. The mean patient age was $48 \pm 14.5$ years (range 28-78 years). The average area of the durotomy (total cortical area mapped) ranged from 26 to $112 \mathrm{~cm}^{2}$ (mean $54.7 \pm 22.3 \mathrm{~cm}^{2}$ ), depending on tumor size. A composite representation of all mapped areas is depicted in Fig. 3.

The final pathologies were as follows: 13 glioblastomas, 5 anaplastic astrocytomas (WHO grade III), 2 diffuse astrocytomas (WHO grade II), 1 low-grade glioma (WHO grade I), 2 oligodendrogliomas (WHO grade II), 1 anaplastic ependymoma (WHO grade III), and 1 lymphoma. Twelve tumors were within the frontal operculum, 5 in the supramarginal gyrus, 2 in the angular gyrus, 3 in the superior temporal gyrus, and 3 in the middle temporal gyrus.

\section{Spatial Overlap of DCS- and TMS-Positive Spots}

Within the respective dural openings, more than twice as many language-positive spots were found on TMS than on DCS (157 vs 74). On DCS, 5.5\% of all stimulated spots were positive (74/1342); on TMS, $12 \%$ were positive. Of the 157 TMS-positive spots, 26 (16.6\%) overlapped with DCS-positive spots (Fig. 1), resulting in a positive predictive value (PPV) of $16.5 \%$ and a high false-positive rate of 83\% (Table 1). Of the 74 DCS-positive spots, 26 (35\%) were TMS copositive. The sensitivity of rnTMS for language was $35 \%$, the specificity was $90 \%$, and the nega- tive predictive value (NPV) was $96 \%$. Since the statistical probability of encountering a language-negative spot at random was $94.5 \%$ (-5.5\% DCS-positive area), rnTMS did not relevantly alter these odds. The positive likelihood ratio (LR+) was 3.5, and the negative likelihood ratio (LR-) was 0.72 .

In $2(8 \%)$ of the 26 DCS/TMS error overlaps, the respective quality of the speech error was congruent. The error was semantic in both cases, and both were in the frontal operculum.

\section{Affiliation to Corina Areas}

In 16 of the 25 patients, TMS- and DCS-positive language spots were found within the same Corina area. In total, 37 of 157 TMS-positive spots were colocalized with DCS-positive spots to the same Corina area. Using this classification, ${ }^{12,23}$ we produced 37 true positives, 120 false positives, 37 false negatives, and 1148 true negatives. These findings then resulted in a PPV of $24 \%$, a sensitivity of $50 \%$, an NPV of $97 \%$, a specificity of $91 \%$, an LR+ of 5.6, and an LR- of 0.55 . In $6(16 \%)$ of the 37 colocalizing spots, the quality of the error was congruent, however; $5(83 \%)$ of the 6 were located in the frontal operculum. The error quality with the most true positives was again semantic.

\section{Anatomical Distribution of DCS- and TMS-Positive Spots}

The overall anatomical distribution of speech-positive spots is depicted in Table 2 and Figs. 2 and 3. The majority of DCS-positive spots could be allocated to the Corina area opercular inferior frontal gyrus (opIFG; 40 spots [54\%]), followed by the triangular inferior frontal gyrus (trIFG; 14 spots [19\%]). The opIFG (pars opercularis) and trIFG (pars triangularis) are part of the frontal operculum and are considered Broca's area in the classic model of language. Taken together, $73 \%$ of language-positive DCS spots were allocated to the classic Broca territory. Eighteen DCS spots $(24 \%)$ were allocated to the supramarginal gyrus (anterior supramarginal gyrus [aSMG], posterior SMG [pSMG]) and the dorsal part of the superior temporal gyrus (middle superior temporal gyrus [mSTG], posterior STG [pSTG]), which is considered the classic Wernicke territory.

TMS-positive spots were more broadly distributed over a larger number of Corina areas. Most TMS-positive spots occurred in the Corina area ventral precentral gyrus (vPrG; 19 spots [12\%]), which is part of the central operculum. On DCS, we produced only dysarthria here; on TMS, however, stimulation of the $\mathrm{vPrG}$ produced various forms of language errors, including semantic errors. If we assigned the TMS-triggered anomias/no responses to dysarthric inhibition (even though on TMS we did not observe the facial/perioral distortions seen on DCS), then TMS produced the most errors in Corina area aSMG (14 spots [9\%]), followed again by the trIFG (12 spots [8\%]).

When we analyzed speech distribution by quality of error, on DCS most anomias occurred in the Corina area opIFG, most semantic errors (by far) in the opIFG, most phonological problems in the opIFG, and most hesitations in the opIFG. On TMS, most anomias were found in the 

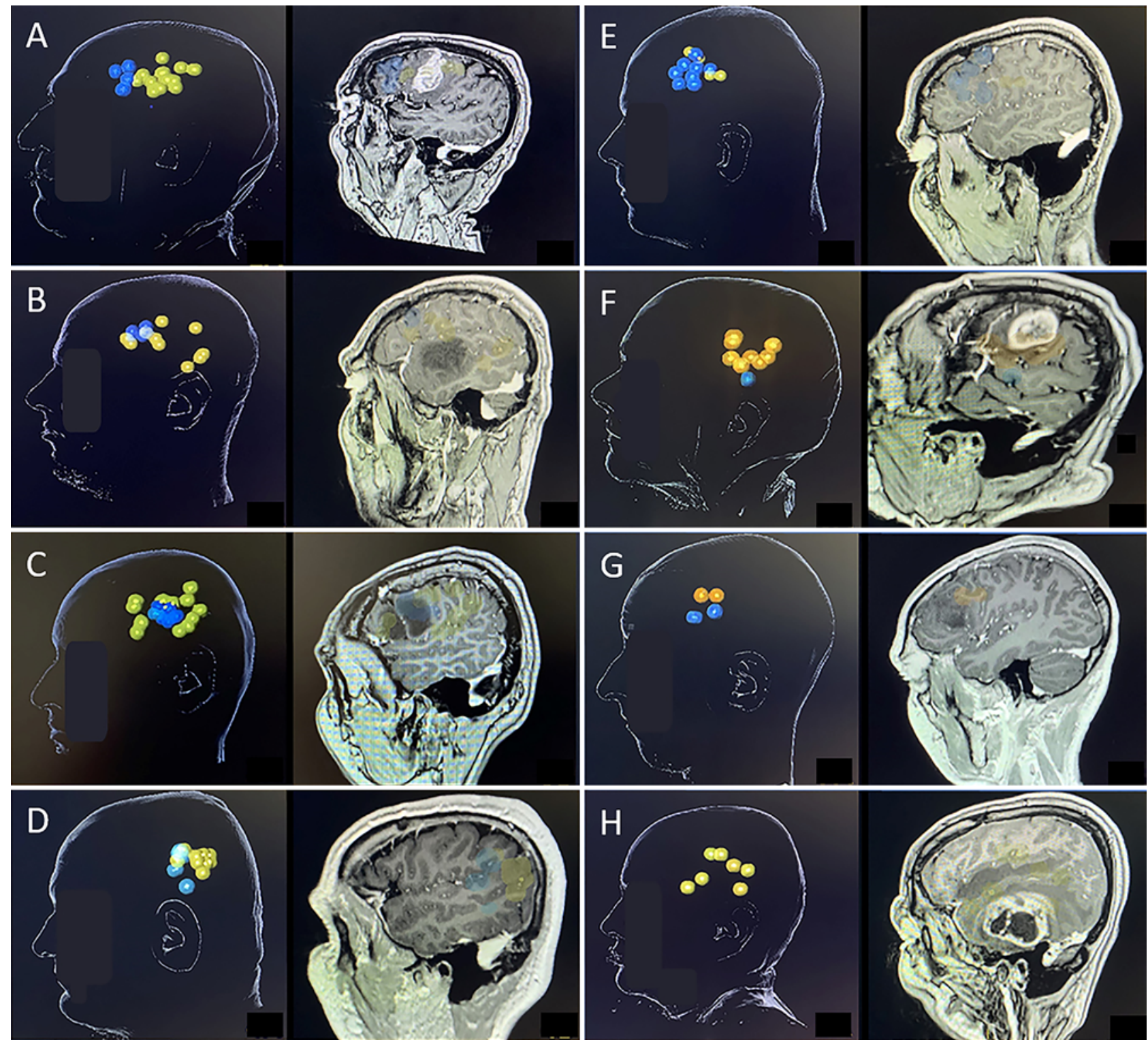

FIG. 1. Coregistration of DCS- and TMS-positive speech spots in 8 patient examples. Blue indicates DCS-positive spots; yellow, TMS-positive spots. DCS and TMS spots were integrated into the same MPRAGE image used for both TMS and intraoperative navigation. Spots were enlarged to $10 \mathrm{~mm}$, representing the assumed electrical field. Each example shows a 3D reconstruction (left) and a sagittal slice (right) through positive spots. Case 1, 1 overlap (A); case 2, 3 overlaps (B); case 3, 2 overlaps (C); case 4 2 overlaps (D); case 5, 3 overlaps (E); case 6, no overlaps (F); case 7, no overlaps (G); case 8, no overlaps (no positive DCS spots within the dural opening, H). Copyright Katharina Faust. Published with permission. Figure is available in color online only.

vPrG (excluding the vPrG, most anomias occurred in the opIFG), most semantic errors were found in the $\mathrm{vPrG}$ and pMFG, most phonological errors occurred in the angular gyrus (anG), and most hesitations were in the aSMG. Overall, the speech quality that occurred most on DCS was no response (32\%), while on TMS it was hesitation (36\%).

Unfortunately, on DCS in 2 patients (resulting in 10 DCS-positive spots total), the quality of linguistic error was not reliably recorded because of a sound-recording problem in our videotape and insufficient paper documentation; therefore, we grouped these errors as "nonclassified" in our analysis.

\section{Discussion}

Previous studies ${ }^{11,12}$ have shown considerably high correlations between DCS- and rnTMS-generated language maps. Our results cannot corroborate these initially promising data. The differences in results can most likely be explained through methodological differences. First, the number of stimulated sites was considerably higher in our cohort (1342 vs 183 in Tarapore et al. ${ }^{11}$ and 160 in Picht et al. ${ }^{12}$ ). This difference may be traced to slightly higher subject numbers, to a potentially denser pattern of stimulation sites, and (probably primarily) to larger craniotomies. 

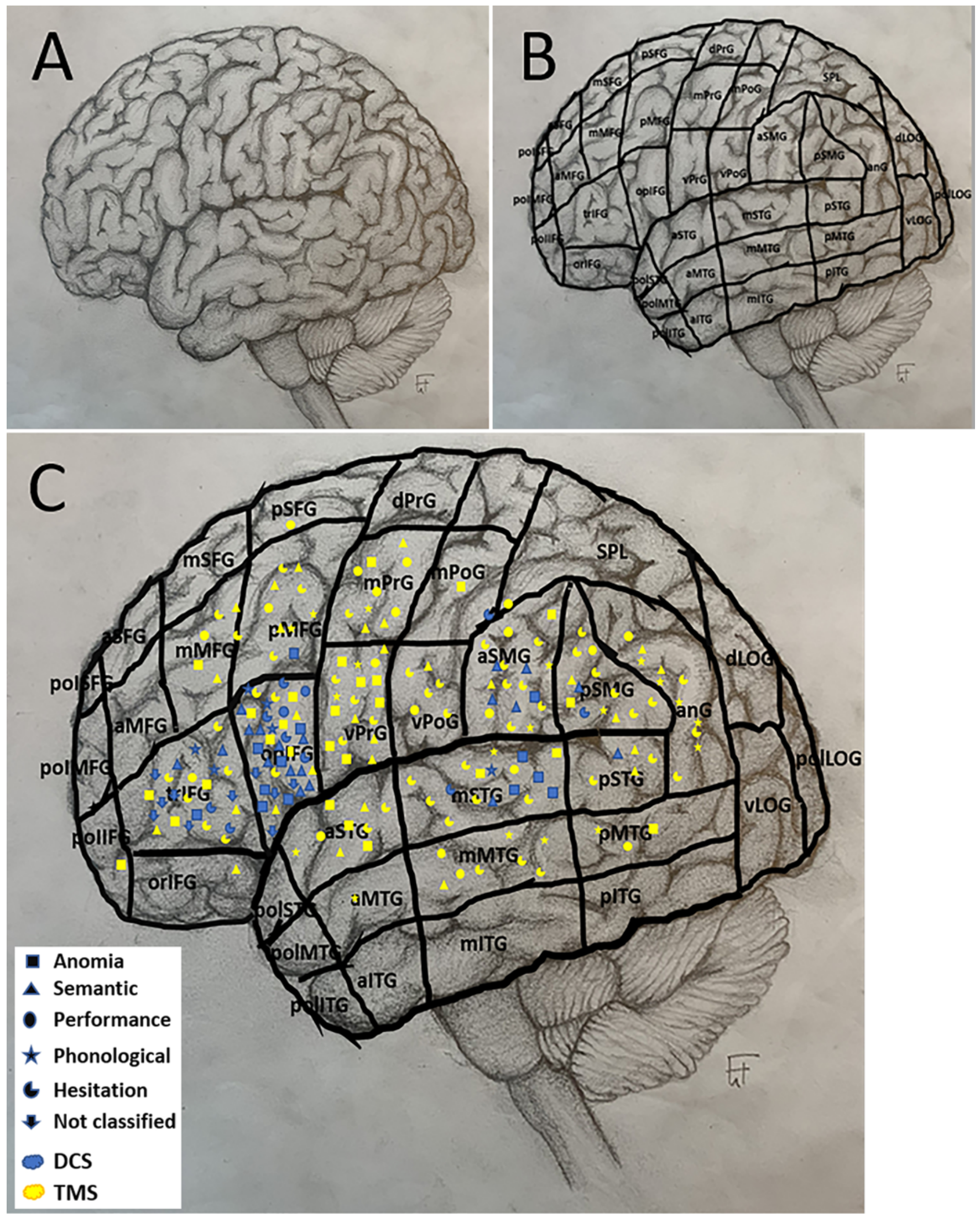

FIG. 2. Allocation of TMS- and DCS-positive language spots to Corina areas. A and B: Parcellation of the human cortex into anatomical areas, as adopted from Corina et al. ${ }^{32} \mathrm{C}$ : Cortical representation of all TMS- and DCS-positive language spots across all subjects with respect to their quality of error and distribution into Corina areas. FIG. 2. (continued) $\rightarrow$ 
FIG. 2. alTG = anterior inferior temporal gyrus; aMFG = anterior middle frontal gyrus; aMTG = anterior middle temporal gyrus; anG = angular gyrus; aSFG = anterior superior frontal gyrus; aSMG = anterior supramarginal gyrus; aSTG = anterior superior temporal gyrus; $\mathrm{dLOG}=$ dorsal lateral occipital gyrus; $\mathrm{dPrG}=$ dorsal precentral gyrus; $\mathrm{mITG}=$ middle inferior temporal gyrus; $\mathrm{mMFG}=$ middle middle frontal gyrus; $\mathrm{mMTG}=$ middle middle temporal gyrus; $\mathrm{mPoG}=$ middle postcentral gyrus; $\mathrm{mPrG}=$ middle precentral gyrus; $\mathrm{mSFG}=$ middle superior frontal gyrus; $\mathrm{mSTG}=$ middle superior temporal gyrus; oplFG = opercular inferior frontal gyrus; orlFG = orbital inferior frontal gyrus; plTG = posterior inferior temporal gyrus; pMFG = posterior middle frontal gyrus; pMTG = posterior middle temporal gyrus; pollFG = polar inferior frontal gyrus; pollTG = polar inferior temporal gyrus; polLOG = polar lateral occipital gyrus; polMFG = polar middle frontal gyrus; polMTG = polar middle temporal gyrus; polSFG = polar superior frontal gyrus; polSTG = polar superior temporal gyrus; $\mathrm{pSFG}=$ posterior superior frontal gyrus; $\mathrm{pSMG}=$ posterior supramarginal gyrus; $\mathrm{pSTG}=$ posterior superior temporal gyrus; $\mathrm{SPL}=$ superior parietal lobe; trIFG = triangular inferior frontal gyrus; $v L O G=$ ventral lateral occipital gyrus; $v P o G=$ ventral postcentral gyrus; $v P r G=$ ventral precentral gyrus. Copyright Katharina Faust. Published with permission. Figure is available in color online only.

Both Picht et al..$^{12}$ and Tarapore et al..$^{11}$ specified their interstimulus distance to be 1 or even $0.5 \mathrm{~cm}$, so the mapped areas were rather small: on average, less than $12 \mathrm{~cm}^{2}$ in Tarapore et al. ${ }^{11}$ and $8 \mathrm{~cm}^{2}$ in Picht et al. ${ }^{12}$ We performed considerably larger craniotomies of $54 \mathrm{~cm}^{2}$ on average. Within those craniotomies, we mapped each square centimeter; mapping larger areas may have led to a preponderance of stimulation-negative sites. Also, in the present cohort we conscientiously aimed to match the number of stimulated sites per area between rnTMS and DCS, with a $1-\mathrm{cm}$ interstimulus distance in both. A mismatch in stimu- lation sites per area may have resulted in systematic bias in the confusion matrix.

Relatively high stimulation intensities for DCS were also used in earlier work. ${ }^{12}$ High stimulation currents may co-stimulate adjacent gyri or even subcortical fiber networks, thus potentially over-representing cortical language sites.

In our present cohort, the rnTMS study and the DCS study were video recorded to allow for retroactive analyses in both modalities. Previous protocols may have had a systematic bias, in which the sensitivity of error record-

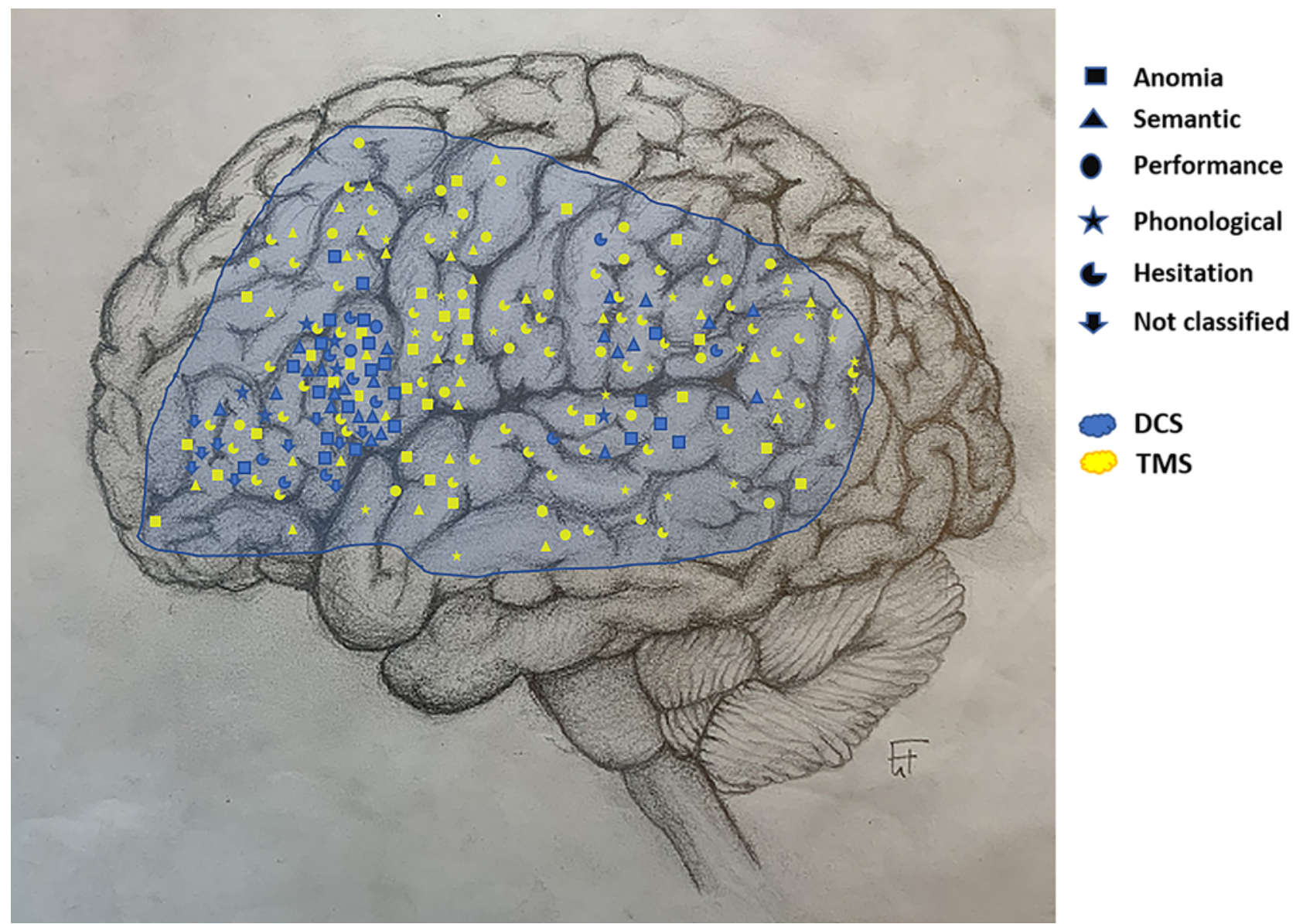

FIG. 3. Composite representation of TMS- and DCS-positive language spots on the cortical surface, a summary of all subjects. Blue-shaded area indicates the sum area of dural openings (mapped areas). Copyright Katharina Faust. Published with permission. Figure is available in color online only. 
TABLE 1. Confusion matrix: distribution of assignments comparing rnTMS language spots with DCS language spots

\begin{tabular}{ccc}
\hline & DCS+ & DCS- \\
\hline rnTMS+ & 26 & 131 \\
\hline rnTMS- & 48 & 1138 \\
\hline
\end{tabular}

The sensitivity of rnTMS for language was $35 \%$, specificity was $90 \%$, NPV was $96 \%$, and PPV was $17 \%$.

ing during DCS may have been potentially lower. More time and ease might have been taken during TMS analysis, while the operating room setting was associated with higher stress levels in all participants, higher levels of background noise and distractors, and time constraints. It is hypothesized that only clear mistakes are recorded in stressful situations, while more subtle mistakes are overlooked. We avoided this systematic bias by having two independent examiners perform ancillary post hoc analyses, for both DCS and TMS.

Previous studies ${ }^{11,12}$ defined affiliation to the same Co- rina area as a true positive, but current language-organization models assume a mosaic-like and hodotopical distribution of essential cortical language sites. ${ }^{33-35}$ Mere coregistration into the same Corina area may not equal functional overlap. Since the Corina areas are relatively large, such analyses undoubtedly produce falsely higher numbers of positive matches. The use of nonuniform and rather discretionary methods of comparing rnTMS to DCS complicated comparisons among previous publications. Finally, the main contributor to the divergent results may be the notable observer-dependent subjectivity in TMS-based language assessment, as reported by Sollman et al. ${ }^{36}$ These shortcomings all indicate that the current TMS protocols used for language detection fall short in terms of reproducibility.

A large number of hesitation errors were recorded in rnTMS in our study; these errors, which have been debated in the literature, ${ }^{28,37}$ may be considered "weak" or "untrustworthy." ${ }^{28}$ Still, most researchers consider them a disruption in word production. If we omitted hesitation errors in our analyses, then the number of rnTMS- and DCS-positive language spots decreased, although congruency did not improve.

TABLE 2. Distribution of DCS- and rnTMS-positive spots with regard to their respective linguistic qualities of error and allocation to Corina areas

\begin{tabular}{|c|c|c|c|c|c|c|c|c|c|c|c|c|c|c|}
\hline \multirow[b]{2}{*}{ Area } & \multicolumn{2}{|c|}{ No Response } & \multicolumn{2}{|c|}{ Semantic } & \multicolumn{2}{|c|}{ Phonological } & \multicolumn{2}{|c|}{ Performance } & \multicolumn{2}{|c|}{ Hesitation } & \multicolumn{2}{|c|}{ Nonclassified } & \multicolumn{2}{|c|}{ Total } \\
\hline & TMS & DCS & TMS & DCS & TMS & DCS & TMS & DCS & TMS & DCS & TMS & DCS & TMS & DCS \\
\hline aMTG & & & & & 1 & & & & & & & & 1 & \\
\hline anG & & & 2 & & 5 & & 1 & & 3 & & & & 11 & \\
\hline aSMG & 1 & 1 & 1 & 5 & 2 & & 3 & & 7 & 1 & & & 14 & 7 \\
\hline aSTG & 3 & & 2 & & 1 & & 1 & & 2 & & & & 9 & \\
\hline mMFG & 1 & 1 & 2 & & & & 1 & & 2 & & & & 6 & 1 \\
\hline mMTG & & & 1 & & 2 & & 2 & & 3 & & & & 8 & \\
\hline $\mathrm{mPoG}$ & 1 & & & & & & & & & & & & 1 & \\
\hline $\mathrm{mPrG}$ & 1 & & 3 & & 1 & & 4 & & 1 & & & & 10 & \\
\hline mSTG & 2 & 4 & & 1 & 1 & 1 & 1 & & 6 & 1 & & & 10 & 7 \\
\hline oplFG & 5 & 14 & 3 & 12 & & 3 & 1 & 2 & 4 & 5 & & 4 & 13 & 40 \\
\hline orlFG & & & 1 & & & & & & & & & & 1 & \\
\hline pMFG & & 1 & 4 & & 2 & & 1 & & 5 & & & & 12 & 1 \\
\hline pMTG & 1 & & & & 1 & & 1 & & & & & & 3 & \\
\hline pollFG & 1 & & & & & & & & & & & & 1 & \\
\hline pSFG & & & & & & & 1 & & & & & & 1 & \\
\hline pSMG & 1 & & 2 & 1 & 1 & & 2 & & 6 & 1 & & & 12 & 2 \\
\hline pSTG & 1 & 1 & 2 & 1 & & & & & 2 & & & & 5 & 2 \\
\hline $\operatorname{trl} I F G$ & 3 & 2 & 2 & 2 & & 2 & 1 & & 6 & 2 & & 6 & 12 & 14 \\
\hline vPoG & & & 1 & & 1 & & 2 & & 4 & & & & 8 & \\
\hline vPrG & 7 & & 4 & & 2 & & 1 & & 5 & & & & 19 & \\
\hline Total & 28 & 24 & 30 & 22 & 20 & 6 & 23 & 2 & 56 & 10 & & 10 & 157 & 74 \\
\hline $\begin{array}{l}\text { aMTG = } \\
\text { mMFG = } \\
\text { gyrus; } m \\
\text { middle fr } \\
\text { pSMG = } \\
\text { postcent }\end{array}$ & $\begin{array}{l}\text { tior mi } \\
\text { dlle mic } \\
=\text { mid } \\
\text { gyrus } \\
\text { terior s } \\
\text { yrus; } v\end{array}$ & $\begin{array}{l}\text { e temp } \\
\text { e fronte } \\
\text { superi } \\
\text { MTG = } \\
\text { camarg }\end{array}$ & $\begin{array}{l}\text { gyrus } \\
\text { rus; } m \\
\text { mpor } \\
\text { terior } \\
\text { gyrus; }\end{array}$ & $\begin{array}{l}\text { anG = } \\
\text { UTG = n } \\
\text { I gyrus; } \\
\text { niddle te } \\
\text { pSTG = }\end{array}$ & $\begin{array}{l}\text { yular gyr } \\
\text { dle midd } \\
\text { IFG = o } \\
\text { poral gy } \\
\text { sterior }\end{array}$ & $\begin{array}{l}\text {; aSMC } \\
\text { tempo } \\
\text { rcular i } \\
\text {; pollF } \\
\text { serior } t\end{array}$ & $\begin{array}{l}\text { = anterio } \\
\text { I gyrus; } \\
\text { rior fror } \\
=\text { polar } \\
\text { poral g. }\end{array}$ & $\begin{array}{l}\text { suprame } \\
\text { PoG = n } \\
\text { ferior fro } \\
\text { us; trlFC }\end{array}$ & $\begin{array}{l}\text { ginal gyr } \\
\text { ddle pos } \\
\text { rlFG }=0 \\
\text { tal gyrus } \\
\text { = triangu }\end{array}$ & $\begin{array}{l}\text { Is; aSTC } \\
\text { central } \\
\text { bital inf } \\
\text { pSFG } \\
\text { ar inferi }\end{array}$ & $\begin{array}{l}=\text { anter } \\
\text { rus; } \mathrm{ml} \\
\text { ior fron } \\
\text { osteric } \\
\text { frontal }\end{array}$ & $\begin{array}{l}\text { superio } \\
=\text { midd } \\
\text { gyrus; } p \\
\text { uperior } \\
\text { rus; } v \mathrm{PP}\end{array}$ & $\begin{array}{l}\text { mporal } \\
\text { precent } \\
F G=p o \\
\text { ntal gyr } \\
=\text { ventr }\end{array}$ & $\begin{array}{l}\text { gyrus; } \\
\text { cal } \\
\text { sterior } \\
\text { us; } \\
\text { al }\end{array}$ \\
\hline
\end{tabular}


Several possible factors could explain why our current rnTMS protocols were insufficient to reliably determine speech-essential areas. First, the neurological system for speech appears far more complex than that for motor functions, so a one-to-one transfer of stimulation protocols may not be feasible. In movement, information is passed on unidirectionally through long fiber tracts that originate in $\mathrm{M} 1$ and terminate in spinal $\alpha$-motoneurons, with some modulation from the supplementary motor area, thalamus, basal ganglia, and cerebellum. In speech production, however, information is likely not unidirectional. ${ }^{38}$ The cortical distribution of sender and recipient neurons may be mosaic-like and closely adjacent, ${ }^{33}$ making a uniform response to electrical current unlikely. In addition, in motor TMS, the stimulation coil is positioned $90^{\circ}$ to the descending fibers. In language TMS, the coil is positioned at various angles to the fibers, at times even parallel. If fiber co-stimulation plays a role in TMS's effect, then the activated tissue may have different modes of transduction into the functional system between language and motor TMS.

Second, the stimulation to locate speech in rnTMS is inhibitory, while the stimulation to locate motor function is excitatory. This disposition has been adopted from DCS, in which similar stimulation parameters can excite movement in M1 and inhibit speech production in languageessential cortices. This transfer may not work in rnTMS, however.

Taken together, our results suggest a relatively high specificity of rnTMS in localizing language-negative areas, yet it fails to pinpoint language-positive areas. One could argue that defining language-negative areas has clinical relevance, since corticotomy can be safely performed through those areas. In our study, however, the craniotomies were relatively large, so sizable numbers of language-negative sites are to be expected statistically. Indeed, only $5.5 \%$ of DCS-stimulated points were language positive, resulting in a $94.5 \%$ chance of encountering a language-negative site at random. rnTMS increased this probability by only $1.5 \%$. The LRs from our data suggest a moderate value for preoperative language rnTMS at most.

\section{Study Limitations and Future Directions}

As with previous studies, our data are subject to the documented poor reproducibility of rnTMS for language, ${ }^{36}$ even though we strove to improve objectivity and validity to some extent by further standardization and employment of an independent linguist. Moreover, navigation-based inaccuracies must be considered: we combined two navigation-based methods, both of which produce an estimated spatial error of 2-3 $\mathrm{mm}$ on their own, $5 \mathrm{~mm}$ or more when combined. Any brain shift after skull opening may further decrease precision.

In order to further improve the validity of rnTMS, repeat postoperative rnTMS mapping in patients who have undergone DCS mapping may help refine technical stimulation parameters. Eventually, only this unique patient group offers verification of true positives. It may also be advisable to omit the $\mathrm{vPrG}$ in future analysis, to avoid confusion with dysarthric inhibition.

\section{Conclusions}

The integration of rnTMS for preoperative noninvasive language mapping is a desirable, yet challenging, concept. While our data revealed high specificities in identifying language-negative areas, the likelihood of detecting language-positive spots was low. When considering rnTMS as a sole source to rely on for preoperative language mapping, our current results are discouraging. Meaningful and dependable stimulation protocols may still need to be established.

\section{References}

1. Frey D, Schilt S, Strack V, et al. Navigated transcranial magnetic stimulation improves the treatment outcome in patients with brain tumors in motor eloquent locations. Neuro Oncol. 2014;16(10):1365-1372.

2. Krieg SM, Shiban E, Buchmann N, et al. Utility of presurgical navigated transcranial magnetic brain stimulation for the resection of tumors in eloquent motor areas. J Neurosurg. 2012;116(5):994-1001.

3. Krieg SM, Sabih J, Bulubasova L, et al. Preoperative motor mapping by navigated transcranial magnetic brain stimulation improves outcome for motor eloquent lesions. Neuro Oncol. 2014;16(9):1274-1282.

4. Picht T, Schmidt S, Brandt S, et al. Preoperative functional mapping for rolandic brain tumor surgery: comparison of navigated transcranial magnetic stimulation to direct cortical stimulation. Neurosurgery. 2011;69(3):581-588.

5. Picht T, Schulz J, Hanna M, et al. Assessment of the influence of navigated transcranial magnetic stimulation on surgical planning for tumors in or near the motor cortex. Neurosurgery. 2012;70(5):1248-1257.

6. Ruohonen J, Karhu J. Navigated transcranial magnetic stimulation. Neurophysiol Clin. 2010;40(1):7-17.

7. Tarapore PE, Picht T, Bulubas L, et al. Safety and tolerability of navigated TMS for preoperative mapping in neurosurgical patients. Clin Neurophysiol. 2016;127(3):1895-1900.

8. Meng Z, Liu S, Zheng Y, Phillips JS. Repetitive transcranial magnetic stimulation for tinnitus. Cochrane Database Syst Rev. 2011;(10):CD007946.

9. Taheri A, Lajevardi M, Arab S, et al. Repetitive transcranial magnetic stimulation for phantom limb pain: probably effective but understudied. Neuromodulation. 2017;20(1):88-89.

10. Manganotti P, Patuzzo S, Cortese F, et al. Motor disinhibition in affected and unaffected hemisphere in the early period of recovery after stroke. Clin Neurophysiol. 2002;113(6):936943.

11. Tarapore PE, Findlay AM, Honma SM, et al. Language mapping with navigated repetitive TMS: proof of technique and validation. Neuroimage. 2013;82:260-272.

12. Picht T, Krieg SM, Sollmann N, et al. A comparison of language mapping by preoperative navigated transcranial magnetic stimulation and direct cortical stimulation during awake surgery. Neurosurgery. 2013;72(5):808-819.

13. Ille S, Sollmann N, Butenschoen VM, et al. Resection of highly language-eloquent brain lesions based purely on rTMS language mapping without awake surgery. Acta Neurochir (Wien). 2016;158(12):2265-2275.

14. Sanai N, Mirzadeh Z, Berger MS. Functional outcome after language mapping for glioma resection. $N$ Engl J Med. 2008;358(1):18-27.

15. Duffau H. Contribution of cortical and subcortical electrostimulation in brain glioma surgery: methodological and functional considerations. Neurophysiol Clin. 2007;37(6):373-382.

16. Spena G, Roca E, Guerrini F, et al. Risk factors for intraop- 
erative stimulation-related seizures during awake surgery: an analysis of 109 consecutive patients. J Neurooncol. 2019;145(2):295-300.

17. Ho AL, Ali R, Connolly ID, et al. Awake versus asleep deep brain stimulation for Parkinson's disease: a critical comparison and meta-analysis. J Neurol Neurosurg Psychiatry. 2018;89(7):687-691.

18. Hervey-Jumper SL, Li J, Lau D, et al. Awake craniotomy to maximize glioma resection: methods and technical nuances over a 27-year period. J Neurosurg. 2015;123(2):325-339.

19. Szelényi A, Bello L, Duffau H, et al. Intraoperative electrical stimulation in awake craniotomy: methodological aspects of current practice. Neurosurg Focus. 2010;28(2):E7.

20. Mikkonen M, Laakso I, Sumiya M, et al. TMS motor thresholds correlate with TDCS electric field strengths in hand motor area. Front Neurosci. 2018;12:426.

21. Nathan SS, Sinha SR, Gordon B, et al. Determination of current density distributions generated by electrical stimulation of the human cerebral cortex. Electroencephalogr Clin Neurophysiol. 1993;86(3):183-192.

22. Parazzini M, Fiocchi S, Chiaramello E, et al. Electric field estimation of deep transcranial magnetic stimulation clinically used for the treatment of neuropsychiatric disorders in anatomical head models. Med Eng Phys. 2017;43:30-38.

23. Ille S, Sollmann N, Hauck T, et al. Combined noninvasive language mapping by navigated transcranial magnetic stimulation and functional MRI and its comparison with direct cortical stimulation. J Neurosurg. 2015;123(1):212-225.

24. Kalbe E, Kessler J, Calabrese P, et al. DemTect: a new, sensitive cognitive screening test to support the diagnosis of mild cognitive impairment and early dementia. Int J Geriatr Psychiatry. 2004;19(2):136-143.

25. Huber W, Poeck K, Willmes K. The Aachen aphasia test. Adv Neurol. 1984;42:291-303.

26. Schwarzer V, Bährend I, Rosenstock T, et al. Aphasia and cognitive impairment decrease the reliability of rnTMS language mapping. Acta Neurochir (Wien). 2018;160(2):343356.

27. Karabanov AN, Raffin E, Siebner HR. The resting motor threshold-restless or resting? A repeated threshold hunting technique to track dynamic changes in resting motor threshold. Brain Stimul. 2015;8(6):1191-1194.

28. Lioumis $\mathrm{P}$, Zhdanov A, Mäkelä N, et al. A novel approach for documenting naming errors induced by navigated transcranial magnetic stimulation. J Neurosci Methods. 2012;204(2):349-354.

29. Ramsay MA, Savege TM, Simpson BR, Goodwin R. Controlled sedation with alphaxalone-alphadolone. BMJ. 1974;2(5920):656-659.

30. Berger MS, Ojemann GA, Lettich E. Neurophysiological monitoring during astrocytoma surgery. Neurosurg Clin $N$ Am. 1990;1(1):65-80.
31. Kombos T, Süss O. Neurophysiological basis of direct cortical stimulation and applied neuroanatomy of the motor cortex: a review. Neurosurg Focus. 2009;27(4):E3.

32. Corina DP, Gibson EK, Martin R, et al. Dissociation of action and object naming: evidence from cortical stimulation mapping. Hum Brain Mapp. 2005;24(1):1-10.

33. Chang EF, Raygor KP, Berger MS. Contemporary model of language organization: an overview for neurosurgeons. $J$ Neurosurg. 2015;122(2):250-261.

34. De Benedictis A, Duffau H. Brain hodotopy: from esoteric concept to practical surgical applications. Neurosurgery. 2011;68(6):1709-1723.

35. Duffau H, Moritz-Gasser S, Mandonnet E. A re-examination of neural basis of language processing: proposal of a dynamic hodotopical model from data provided by brain stimulation mapping during picture naming. Brain Lang. 2014;131:1-10.

36. Sollmann N, Hauck T, Hapfelmeier A, et al. Intra- and interobserver variability of language mapping by navigated transcranial magnetic brain stimulation. BMC Neurosci. 2013; $14: 150$.

37. Schuhmann T, Schiller NO, Goebel R, Sack AT. Speaking of which: dissecting the neurocognitive network of language production in picture naming. Cereb Cortex. 2012;22(3):701709.

38. Ries SK, Piai V, Perry D, et al. Roles of ventral versus dorsal pathways in language production: an awake language mapping study. Brain Lang. 2019;191:17-27.

\section{Disclosures}

The authors report no conflict of interest concerning the materials or methods used in this study or the findings specified in this paper.

\section{Author Contributions}

Conception and design: Faust. Acquisition of data: Faust, Bährend, Muench, Schneider, Moshourab. Analysis and interpretation of data: Faust, Bährend, Dreyer. Drafting the article: Faust. Critically revising the article: Bährend, Moshourab, Dreyer, Vajkoczy, Picht. Reviewed submitted version of manuscript: Faust, Bährend, Schneider, Moshourab, Dreyer, Vajkoczy, Picht. Approved the final version of the manuscript on behalf of all authors: Faust. Statistical analysis: Faust. Study supervision: Faust, Picht.

\section{Correspondence}

Katharina Faust: Charité Universitätsmedizin Berlin, Germany. katharina.faust@charite.de. 